\title{
Bipartite Crossing Numbers of Meshes and Hypercubes
}

\author{
Farhad Shahrokhi ${ }^{1, \star}$, Ondrej Sýkora ${ }^{2, \star \star}$, László A. Székely ${ }^{3, \star}$, Imrich Vrto ${ }^{2}$ \\ 1 Department of Computer Science, University of North Texas \\ P.O.Box 13886, Denton, TX 76203-3886, USA \\ 2 Institute for Informatics, Slovak Academy of Sciences \\ P.O.Box 56, 840 00 Bratislava, Slovak Republic \\ 3 Department of Mathematics, University of South Carolina \\ Columbia, SC 29208, USA
}

\begin{abstract}
Let $G=\left(V_{0}, V_{1}, E\right)$ be a connected bipartite graph, where $V_{0}, V_{1}$ is the bipartition of the vertex set $V(G)$ into independent sets. A bipartite drauing of $G$ consists of placing the vertices of $V_{0}$ and $V_{1}$ into distinct points on two parallel lines $x_{0}, x_{1}$, respectively, and then drawing each edge with one straight line segment which connects the points of $x_{0}$ and $x_{1}$ where the endvertices of the edge were placed. The bipartite crossing number of $G$, denoted by ber $(G)$ is the minimum number of crossings of edges over all bipartite drawings of $G$. We develop a new lower bound method for estimating $b \mathrm{cr}(G)$. It relates bipartite crossing numbers to edge isoperimetric inequalities and Laplacian eigenvalues of graphs. We apply the method, which is suitable for "well structured" graphs, to hypercubes and 2-dimensional meshes. E.g. for the $n$-dimensional hypercube graph we get $n 4^{n-2}-O\left(4^{n}\right) \leq b c r\left(Q_{n}\right) \leq n 4^{n-1}$. We also consider a more general setting of the method which uses eigenvalues, but as a trade-off for generality, often gives weaker results.
\end{abstract}

\section{Introduction}

Let $G=\left(V_{0}, V_{1}, E\right)$ be a connected bipartite graph, where $V_{0}, V_{1}$ is the bipartition of vertices into independent sets. A bipartite drawing of $G$ consists of placing the vertices of $V_{0}$ and $V_{1}$ into distinct points on two parallel lines $x_{0}, x_{1}$, respectively, and then drawing each edge with one straight line segment which connects the points of $x_{0}$ and $x_{1}$ where the endvertices of the edge were placed. The bipartite crossing number of $G$, denoted by $b c r(G)$ is the minimum number

* The research of the first author was supported in part by the NSF grant CCR 9528228.

** The research of the 2nd and the 4 th author was partially supported by the Alexander von Humboldt Foundation, by the Slovak Scientific Grant Agency grant No. 95/5305/277 and by grant of EU INCO-COP 96-0195.

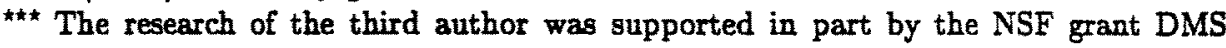
9701211 and the Hungarian NSF grants T 016358 and T 019367. 
of crossings of edges over all bipartite drawings of $G$. The bipartite crossing number is one of the parameters which strongly influences the aesthetics of drawings of hierarchical graphs.

The problem of finding the bipartite crossing number is a variant of the standard crossing number problem, see e.g. [21], and was first introduced by Harary [13] and Harary and Schwenk [14]. In [14], they proved bcr $(G)=0$ iff $G$ is a caterpillar. Further, they obtained the exact values of the bipartite crossing numbers of subdivisions of complete and complete bipartite graphs. For even cycles they showed bcr $\left(C_{3 n}\right)=n-1$. The bipartite crossing number problem was also proposed by Watkins [26] independently. Some basic observations on $b c r(G)$ were made by May and Szkatula [19]. The bipartite crossing number problem is known to be NP-complete [12] but can be solved in polynomial time for bipartite permutation graphs [22], and trees [20]. A great deal of research has been devoted to the design of algorithms and heuristics for solving this problem $[7,11,16,23,25]$. Brandenburg, Jünger und Mutzel [6] have called for some entirely new approaches because the usual heuristics do not give good results for the bipartite crossing number. The latest progress in this area was made in [20] in which we have shown an intimate relationship between the bipartite crossing number problem and the optimal linear arrangement problem. These result have led to the first provably good approximation algorithms for computing $b c r(G)$. The restricted problem when the positions of the vertices of $V_{0}$ are given is also NP-complete [11] and frequently appears in drawing of hierarchical graphs [7, 25], see also the survey [10]. Eades and Wormald [11] designed a polynomial time algorithm which approximates the bipartite crossing number within a multiplicative factor of 3 in this restricted problem.

In this paper we develop a new lower bound argument which is suitable for "well structured graphs". The argument relates the bipartite crossing number of a graph to the edge isoperimetric inequality of the graph. We apply our new lower bound technique for two instances of important graphs: hypercubes and two-dimensional meshes. We obtain here substantially better lower bounds on the bipartite crossing number of theses graphs than the bounds that one can obtain by using the general results in [20]. For the meshes we get $\frac{3}{4} m^{2} n-\frac{1}{4} m^{3}-\frac{9}{2} m n-$ $\frac{3}{2} m^{2}-3 m \leq b c r(M(m, n)) \leq \frac{3}{2} m^{2} n$, and for the $n$-dimensional hypercube graph we get $n 4^{n-2}-O\left(4^{n}\right) \leq b c r\left(Q_{n}\right) \leq n 4^{n-1}$. It is worth mentioning that especially for the $3 \times n$ mesh $M(3, n)$ we get an exact result $b c r(M(3, n))=5 n-6$.

We close the paper by describing a general framework to give lower bounds for bipartite crossing numbers using Laplacian eigenvalues and we develop our ideas on the two examples.

\section{Notations and Definitions}

Let $\delta$ and $\Delta$ denote minimum and maximum degrees of $G$, respectively. The degree of vertex $v$ will be denoted by $d(v)$. For $X \subseteq V(G)$, let vol $(X)$ denote $\sum_{v \in X} d(v)$. For a bipartite drawing $D(G)$ of a graph $G$, let $b c r(D(G))$ denote the number of the crossings in $D(G)$ (i.e. the number of unordered pairs of 
crossing edges), and define the bipartite crossing number of $G$ by $b c r(G)=$ $\min _{D(G)} b c r(D(G))$.

Given an arbitrary graph $G=(V, E)$, and a bijection $F: V \rightarrow\{1,2,3, \ldots,|V|\}$, define the length of $F$, as $\sum_{u v \in E}|F(u)-F(v)|$. The optimal linear arrangement problem is to find a bijection $F$, of minimum length. This minimum value is denoted by $L(G)$, the optimal linear arrangement value of $G$.

For $X \subseteq V$ define

$$
\partial(X)=\{u v \in E: u \in X, v \in V-X\} .
$$

We call $\partial(X)$ the edge boundary of $X$. The general objective is to find a good approximating and easily described real function $f(x)$ such that $|\partial(X)| \geq f(|X|)$, for all $X \subseteq V(G)$. Such an inequality is called an edge isoperimetric inequality $[2,3]$; edge isoperimetric inequalities have several applications in graph theory and computer science.

For $X, Y \subset V$ define cut $(X, Y)$ to be a smallest size set of edges in $G$ which separates $X$ from $Y$ in $G$. Note that $|\operatorname{cut}(X, Y)| \leq|\partial(X)|$.

For $m \leq n$, let $M(m, n)$ denote the 2-dimensional mesh i.e. the graph defined by the Cartesian product of an $m$-vertex path with an $n$-vertex path. Let $Q_{n}$ denote the $n$-dimensional hypercube graph, i.e. the Cartesian product of $n 2$ vertex paths.

\section{Meshes}

In [20] we have shown that

$$
\frac{\delta}{96} L(G) \leq b c r(G) \leq \Delta L(G),
$$

for $\delta \geq 2$ (the lower bound holds for $|E(G)| \geq 1.05|V(G)|$ and in [20] the constant in the lower bound actually was $1 / 60$ ). By composing our formula (1) with a result from [18]:

$$
L(M(m, n))=\left(m^{2} n-\frac{\sqrt{2}-1}{3} m^{3}\right)+O(m n),
$$

we get lower and upper bounds for $b c r(M(m, n))$. The issue here is to improve these bounds by constant multiplicative factors.

Now we will improve the upper bound by a "column after column" drawing and the lower bound by a new lower bound argument.

Theorem 3.1 For a mesh $M(m, n), 4 \leq m \leq n$ it holds:

$$
\frac{3}{4} m^{2} n-\frac{1}{4} m^{3}-\frac{7}{2} m n-\frac{1}{2} m^{2}+6 n+\frac{9}{2} m \leq b c r(M(m, n)) \leq \frac{3}{2} m^{2} n .
$$


Proof. Upper bound. First place the vertices of $M(m, n)$ on the line $x_{0}$ by the column after column manner. Then project the vertices of $V_{1}$ on $x_{1}$. The result of the theorem follows by counting the crossings between every two consecutive columns. Their number equals $\left(3 m^{2}-7 m+4\right) / 2$.

Lower bound. For the sake of simplicity assume that both $m$ and $n$ are even. Consider a bipartite drawing of $M(m, n)$. Then $\left|V_{0}\right|=m n / 2$. For $k=1,2, \ldots, m n / 2$, let $A_{k}$ denote the set of the first $k$ vertices on $x_{0}$ from the left. A variant of the Menger's theorem [24] says that the maximum number of edge disjoint paths between $A_{k}$ and $V_{0}-A_{k}$ equals $\left|\operatorname{cut}\left(A_{k}, V_{0}-A_{k}\right)\right|$.

Claim. Each of these paths, except for those ending in the $(k+1)$-st vertex $v_{k+1}$ on $x_{0}$ from the left, must cross all but one edges adjacent to the $(k+1)$-st vertex $v_{k+1}$.

Define a function

$$
f(x)=\left\{\begin{array}{l}
2 \sqrt{x}, \text { if } 0 \leq x \leq m^{2} / 4, \\
m, \text { if } m^{2} / 4 \leq x \leq m n-m^{2} / 4, \\
2 \sqrt{m n-x}, \text { if } m n-m^{2} / 4 \leq x \leq m n .
\end{array}\right.
$$

Now we use an edge isoperimetric inequality for meshes. It is known $[1,4]$ that for any $X \subset M(m, n),|\partial(X)| \geq f(|X|)$ holds. The set $\operatorname{cut}\left(A_{k}, V_{0}-A_{k}\right)$ partitions $V(G)$ into $X$ and $V(G)-X$ such that $A_{k} \subset X$ and $V_{0}-A_{k} \subset V(G)-X$. (Actually $X=X\left(A_{k}\right)$ depends on $A_{k}$, but for the sake of simplicity we do not show this dependence in our notation.) Clearly $\left|\operatorname{cut}\left(A_{k}, V_{0}-A_{k}\right)\right|=|\partial(X)|$. As $A_{k} \subset X \subset V(G)-\left(V_{0}-A_{k}\right)$, the concavity of $f$ gives

$\left|\operatorname{cut}\left(A_{k}, V_{0}-A_{k}\right)\right| \geq \min \left\{f\left(\left|A_{k}\right|\right), f\left(\left|V(G)-\left(V_{0}-A_{k}\right)\right|\right)\right\}=\min \left\{f(k), f\left(\frac{m n}{2}+k\right)\right\}$.

There are at most $m+n$ vertices in $V_{0}$ whose degree is less than 4 . We are going to give a lower bound for the number of crossings that happen to edges adjacent to $v_{k+1}$. It is convenient not counting the contribution of vertices $v_{k+1}$ whose degree is less than 4. Hence if $k$ runs from 1 to $m n / 2-1$, using only vertices $v_{k+1}$ whose degree is 4 , the Claim yields that all $\left(A_{k}, V_{0}-A_{k}\right)$ paths but 4 intersect at least 3 of the edges adjacent to $v_{k+1}$. Below a sum with a prime indicates $m+n$ missing terms. We obtain

$$
\operatorname{bcr}(M(m, n)) \geq \frac{3}{2} \sum_{k=1}^{\frac{m^{n}-1}{2}} \prime\left(\left|c u t\left(A_{k}, V_{0}-A_{k}\right)\right|-4\right)
$$

The denominator 2 occurs before the sum is because each crossing is counted at most twice. Further,

$$
\begin{aligned}
\operatorname{bcr}(M(m, n)) & \geq \frac{3}{2} \sum_{k=1}^{\frac{m a}{2}-1}\left\{\min \left\{f(k), f\left(\frac{m n}{2}+k\right)\right\}-3 m n\right. \\
& =\frac{3}{2} \sum_{k=1}^{\frac{m^{3}}{4}} \min \left\{f(k), f\left(\frac{m n}{2}+k\right)\right\}+\frac{3}{2} \sum_{k=\frac{m^{2}}{4}+1}^{\frac{m n}{2}-\frac{m^{2}}{4}-1} / \min \left\{f(k), f\left(\frac{m n}{2}+k\right)\right\}
\end{aligned}
$$




$$
\begin{aligned}
& +\frac{3}{2} \sum_{k=\frac{m n}{2}-m^{2}}^{\frac{m n}{2}-1} \min \left\{f(k), f\left(\frac{m n}{2}+k\right)\right\}-3 m n \\
& \geq 6 \sum_{k=1}^{\frac{m^{2}}{4}} \sqrt{k}+\frac{3}{4}\left(m^{2} n-m^{3}-4 m\right)-3 m n-(m+n) \frac{3 m}{2} \\
& \geq 6 \int_{0}^{\frac{m^{2}}{4}} \sqrt{x} d x+\frac{3}{4}\left(m^{2} n-m^{3}-4 m\right)-3 m n-(m+n) \frac{3 m}{2} \\
& \geq \frac{3}{4} m^{2} n-\frac{1}{4} m^{3}-\frac{9}{2} m n-\frac{3}{2} m^{2}-3 m .
\end{aligned}
$$

We excluded from Theorem 3.1 the cases $m=2,3$. The result $b c r(M(2, n))=$ $n-1$ can be easily deduced from the optimal bipartite drawing of the even cycle $C_{2 n},[14]$.

Theorem 3.2 For $n \geq 3$ it holds:

$$
\operatorname{bcr}(M(3, n))=5 n-6 .
$$

Proof. Upper bound. First place all vertices of $M(3, n)$ on $x_{0}$ in a column after column manner. Then project the vertices of $V_{1}$ on $x_{1}$. It is easy to see by induction on $n$ that the resulting drawing has $5 n-6$ crossings.
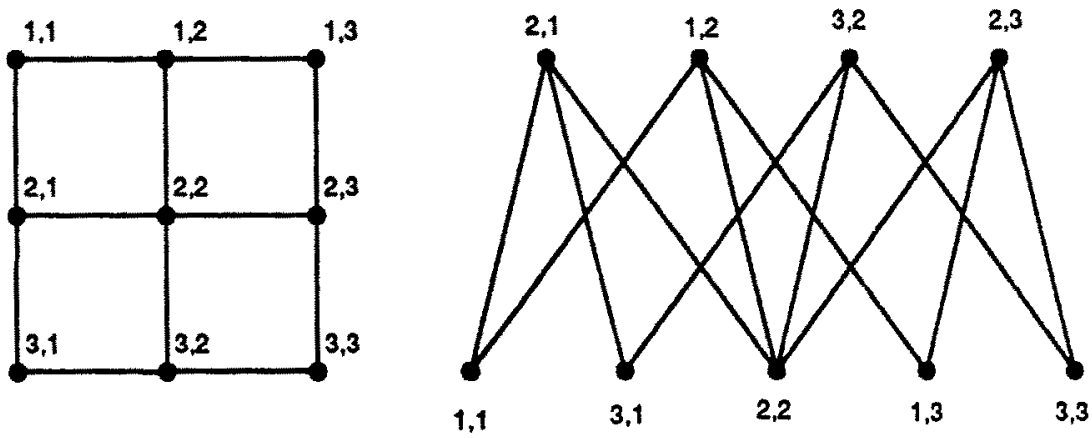

Fig. 1: Mesh $M(3,3)$ and its optimal bipartite drawing

Lower bound. Imagine that $M(3, n)$ consists of 3 row and $m$ column vertices. Let $M(3,3)$ denote the submesh induced by the last 3 column vertices. We proceed by induction on $n$. By a case analysis we can show that $b c r(M(3,3))=9$. Suppose that $\operatorname{bcr}(M(3, n-1)) \geq 5(n-1)-6$, for $n \geq 4$ and consider $M(3, n)$. Using a case analysis again one can show that the edges incident to the last column vertices in $M(3, n)$ contain at least 5 crossings. In fact this can be verifed considering the submesh $M(3,3)$ only. Therefore

$\operatorname{bcr}(M(3, n)) \geq \operatorname{bcr}(M(3, n-1))+5 \geq 5 n-6$. 


\section{Hypercubes}

For hypercubes we could apply again our result (1) from [20] and compose it with a result of Harper who showed in [15] that: $L\left(Q_{n}\right)=2^{n-1}\left(2^{n}-1\right)$. This would give us lower and upper bounds on $b c r\left(Q_{n}\right)$. The issue here is to obtain better bounds than those. The upper bound is given by a recursive drawing. Then we adjust the lower bound argument from the previous section to hypercubes.

Theorem 4.1 For $n \geq 3$ it holds:

$$
n 4^{n-2}-O\left(4^{n}\right)<\operatorname{bcr}\left(Q_{n}\right) \leq n 4^{n-1}
$$

Proof. Upper bound. Draw $Q_{n}$ recursively. The drawing of $Q_{2}$ is unique. Assume that we have a drawing $D\left(Q_{n-1}\right)$ with

$$
b c r\left(D\left(Q_{n-1}\right)\right) \leq(2 n-5) 2^{2 n-5}-\left((n-1)^{2}-(n-1)-1\right) 2^{n-3} .
$$

Place a copy of the drawing $D\left(Q_{n-1}\right)$ next to the drawing $D\left(Q_{n-1}\right)$ and add $2^{n-1}$ new edges to complete the drawing of $D\left(Q_{\pi}\right)$. There are $2^{2 n-4}$ crossings of new edges with new edges and $(n-1) 2^{n-1}\left(2^{n-2}-1\right)$ crossings of new edges with old edges. See Fig. 2. We have:

$$
\begin{aligned}
b c r\left(D\left(Q_{n}\right)\right) & \leq 2 b c r\left(D\left(Q_{n-1}\right)\right)+2^{2 n-4}+(n-1) 2^{n-1}\left(2^{n-2}-1\right) \\
& =(2 n-3) 2^{2 n-3}-\left(n^{2}-n-1\right) 2^{n-2}<n 4^{n-1} .
\end{aligned}
$$
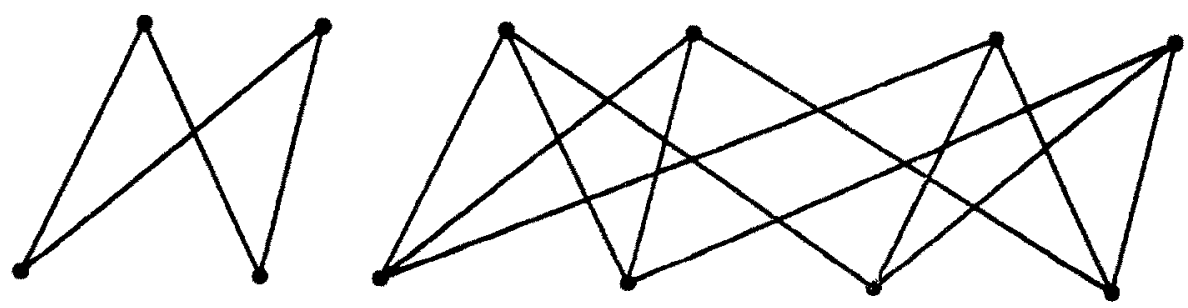

Fig. 2: Bipartite drawings of $Q_{2}$ and $Q_{3}$.

Lower bound. We apply the same argument as for 2-dimensional meshes. Consider a bipartite drawing of $Q_{n}$. Note that $\left|V_{0}\right|=2^{n-1}$. For $k=1,2, \ldots, 2^{n-1}-1$, let $A_{k} \subset V_{0}$ denote the set of the first $k$ vertices on $x_{0}$ from the left. Following Bollobás and Leader [5] define a function $f(x)$ as follows:

$$
f(x)=\left\{\begin{array}{l}
x(n-\log x), \text { if } 1 \leq x \leq 2^{n-1}, \\
\left(2^{n}-x\right)\left(n-\log \left(2^{n}-x\right)\right), \text { if } 2^{n-1} \leq x \leq 2^{n} .
\end{array}\right.
$$

(Here log denotes logarithm based 2.) An edge isoperimetric inequality for hypercubes (see e.g [9]) says that for any $X \subset Q_{n}$, the inequality $|\partial(X)| \geq f(|X|)$ holds. Following the reasoning applied for meshes (i.e. $\left|\operatorname{cut} t\left(A_{k}, V_{0}-A_{k}\right)\right| \geq$ $\min \left(f(k), f\left(2^{n-1}+k\right)\right)$ for $\left.1 \leq k \leq 2^{n-1}\right)$ we show that

$$
\left|c u t\left(A_{k}, V_{0}-A_{k}\right)\right| \geq \min \left\{k(n-\log k),\left(2^{n-1}-k\right)\left(n-\log \left(2^{n-1}-k\right)\right)\right\} .
$$


Hence if $k$ runs from 1 to $2^{n-1}-1$ we get

$$
\begin{aligned}
& b c r\left(Q_{n}\right) \\
\geq & \frac{n-1}{2} \sum_{k=1}^{2^{n-1}-1}\left(\left|\operatorname{cut}\left(A_{k}, V_{0}-A_{k}\right)\right|-n\right) \\
\geq & \frac{n-1}{2} \sum_{k=1}^{2^{n-1}-1} \min \left\{k(n-\log k),\left(2^{n-1}-k\right)\left(n-\log \left(2^{n-1}-k\right)\right)\right\}-n(n-1) 2^{n-2} \\
\geq & (n-1) \sum_{k=1}^{2^{n-2}-1} k(n-\log k)+(n-1) 2^{n-2}-n(n-1) 2^{n-2} \\
= & n(n-1) 2^{n-3}\left(2^{n-2}-1\right)+(n-1) 2^{n-2}-n(n-1) 2^{n-2}-(n-1) \sum_{k=1}^{2^{n-2}-1} k \log k,
\end{aligned}
$$

where we used that for $k \leq 2^{n-2}$ it holds $k(n-\log k) \leq\left(2^{n-1}-k\right)\left(n-\log \left(2^{n-1}-\right.\right.$ $k)$ ). Observe that

$$
\sum_{k=1}^{2^{n-2}-1} k \log k<\int_{1}^{2^{n-2}} x \log x d x=(n-2) 2^{2 n-5}-\frac{1}{\ln 2} 2^{2 n-6}+\frac{1}{4 \ln 2} .
$$

Substituting this into the previous inequality we get the result.

\section{A general lower bound method based on eigenvalues}

Our basic reference to spectral graph theory is Fan Chung's recent book [8]. We use Laplacian eigenvalues of a graph like [8] and define $\lambda_{G}$ as the smallest positive Laplacian eigenvalue of the graph $G$.

The connection between eigenvalues and isoperimetric inequalities has been subject of study since long. We recall the following theorem from Section 2.2 of [8]: For $X \subseteq V(G)$

$$
|\partial X| \geq \frac{\lambda_{G}}{2} \min (\operatorname{vol}(X), \operatorname{vol}(V(G)-X)) .
$$

Assume now $G=\left(V_{0}, V_{1}, E\right)$ is a bipartite graph in an optimal bipartite drawing $D$. We apply to $b c r(G)$ the lower bound technique developed for hypercubes and meshes. Let $v_{i}$ denote the $i$-th vertex in $V_{0}$ and $A_{i}$ denotes the set of the first $i$ vertices in $V_{0}$.

$$
\begin{aligned}
\left|\operatorname{cut}\left(A_{i}, V_{0}-A_{i}\right)\right| & =\left|\partial X\left(A_{i}\right)\right| \geq \frac{\lambda_{G}}{2} \min \left(\operatorname{vol}\left(X\left(A_{i}\right)\right), \operatorname{vol}\left(V(G)-X\left(A_{i}\right)\right)\right)(3) \\
& \geq \frac{\lambda_{G}}{2} \min \left(\operatorname{vol}\left(A_{i}\right), \operatorname{vol}\left(V_{0}-A_{i}\right)\right)
\end{aligned}
$$


Using (2) for estimating $|\partial X|$, instead of an explicit function $f(x)$ that is rarely known, we end up with the estimate

$$
2 b c r(G) \geq \sum_{i=1}^{\left|V_{0}\right|-1}\left(d\left(v_{i+1}\right)-1\right)\left(\frac{\lambda_{G}}{2} \min \left(\operatorname{vol}\left(A_{i}\right), \operatorname{vol}\left(V_{0}-A_{i}\right)\right)-d\left(v_{i+1}\right)\right) .
$$

Formula (5) applies very easily when all degrees in $V_{0}$ are the same. This is the case, for example, for hypercubes. In this case $\lambda_{Q_{n}}=2 / n$ (p. 6 in [8]) and we easily can derive the lower bound of our Theorem 4.1 with a slightly weaker (halved) multiplicative constant.

It is instructive to see how far can we get with eigenvalues if we try this approach to meshes. The first problem that we face is that the graph is not regular. It is unclear how to give good lower bounds for $\sum_{i} a_{i} b_{i}$ in the RHS of (5) in general. This problem can be overcome since almost all degrees are 4 if both $n, m$ grow large.

The bigger problem is that $\lambda_{M(n, m)}$ is simply not large enough. The smallest positive Laplacian eigenvalue of an $n$-vertex path is $1-\cos \frac{\pi}{n-1}=\theta\left(\frac{1}{n^{2}}\right)$ [8], p. 6. By the results of [8], p. $37, \lambda_{M(n, m)}=\theta\left(1-\cos \frac{\pi}{n-1}\right)=\theta\left(\frac{1}{n^{2}}\right)$, since $n \geq m$. Therefore in this way we cannot get anything as good as our lower bound in Theorem 3.1.

Note that [17] has developed a lower bound for the linear arrangement value of a graph in terms of the smallest positive Laplacian eigenvalue. This lower bound can be combined with (1) to obtain a bound (5). However, due to the large constant in (1), formula (5) is expected to give tighter bounds.

\section{Concluding Remarks}

We introduced a new method for establishing lower bounds on bipartite crossing numbers. The method is based on edge isoperimetric inequalities. By means of the new method we essentially improved the previous lower bounds for meshes and hypercubes. The method is especially well suited for "well structured graphs", for which good edge isoperimetric inequalities and/or the smallest positive Laplacian eigenvalue are known [2]. Our upper and lower bounds still leave space for possible improvements. We believe that the upper bounds are closer to the optimal values than the lower bounds.

Acknowledgment. The research was partly done while the second and fourth authors were visiting Department of Mathematics and Informatics of University in Passau. They thank Prof. F.-J. Brandenburg for invitation and for the inspiring atmosphere in his section.

The figures were produced by system INCA of Olivier Boudcn, LaBRI, Bordeaux I.

\section{References}

1. Ahlswede, R., Berrukov, S. L., Edge isoperimetric theorems for integer point arrays, Appl. Math. Lett. 8 (1995), 75-80. 
2. Bezrukov, S. Ln, Edge isoperimetric problems on graphs, Technical Report, Department of Computer Science, University of Paderborn, 1997.

3. Bollobás, B., Combinatorics, Chapter 16, Cambridge Uni. Press, 1986.

4. Bollobás, B., Leader, I., Edge-isoperimetric inequalities in the grid, Combinatorica 11 (1991), 299-314.

5. Bollobás, B., Leader, I., Matchings and paths in cubes, SIAM J. Discrete Mathematics, to appear.

6. Brandenburg, F. J., Jünger, M., Mutzel, P., Algorithms for automatic graph drawing, Technical Report, Max Planck Institute, MPI-1-97-1-007, Saarbrücken, March 1997, (in German).

7. Catarci, T., The assignment heuristics for crossing reduction, IEEE Transactions on Systems, Man and Cybernetics 25 (1995), 515-521.

8. Chung, F. R. K., Spectral Graph Theory, Regional Conference Series in Mathematics Number 92, American Mathematical Society, Providence, RI, 1997.

9. Chung, F. R. K., Füredi, Z., Graham, R. L., Seymour, P. D., On induced subgraphs of the cube, $J$. Combinatorial Theory (A) 48 (1988), $180-187$.

10. Di Battista, J., Eades, P., Tamassia, R., Tollis, I.G., Algorithms for drawing graphs: an annotated bibliography, Computational Geometry 4 (1994), 235-282.

11. Eades, P., Wormald, N., Edge crossings in drawings of bipartite graphs, Algorithmica 11 (1994), 379-403.

12. Garey, M.R., Johnson, D.S., Crossing number is NP-complete, SIAM J. Algebraic and Discrete Methods 4 (1983), 312-316.

13. Harary, F., Determinants, permanents and bipartite graphs, Mathematical Magazine 42 (1969), 146-148.

14. Harary, F., Schwenk, A., A new crossing number for bipartite graphs, Utilitas Mathematica 1 (1972), 203-209.

15. Harper, L. H., Optimal assignements of numbers to vertices, SIAM J. Applied Mathematics 12 (1964), 131-135.

16. Jünger, M., Mutzel, P., Exact and heuristic algorithm for 2-layer straightline crossing number, in: Proc. Graph Drawing'95, Lecture Notes in Computer Science 1027, Springer Verlag, Berlin, 1996, 337-348.

17. Juvan, M., Mohar, B., Optimal linear labelings and eigenvalues of graphs, Discrete Applied Mathematics 36 (1992), 153-168.

18. Muradyan, D.O., Piliposian, T.E., Minimal numberings of vertices of a rectangular lattice, Akad. Nauk Armjan. SSR Doklady 70 (1980), 21-27, (in Russian).

19. May, M., Szlatula, K., On the bipartite crossing number, Control and Cybernetics 17 (1988), 85-98.

20. Shahrokhi, F., Sýkora, O., Székely, L. A., Vrio, On bipartite crossings, biplanar subgraphs, and the linear arrangement problem, in: Proc. 5th Workshop Algorithms and Data Structures, (WADS'97), August 6-8, 1997 Halifax, Nova Scotia, Canada, Lecture Notes in Computer Science Vol. 1272, Springer-Verlag, 55-68.

21. F. Shahrokhi, L. A. Székely, I. Vrt'o, Crossing numbers of graphs, lower bound techniques and algorithms: a survey, in: Proc. DIMACS Workshop on Graph Drouing'94, Lecture Notes Computer Science 894, Springer Verlag, Berlin, 1995, 131142.

22. Spinrad, J., Brandstädt, A., Stewart, L., Bipartite permutation graphs, Discrete Applied Mathematics 18, 1987, 279-292.

23. Sugiyama, K., Tagawa, S., Toda, M., Methods for visual understanding of hierarchical systems structures, IEEE Transactions on Systems, Man and Cybernetics 11 (1981), 109-125. 
24. Tutte, W., T., Graph Theory, Addison-Wesley Publishing Company, Reading, 1984.

25. Warfield, J., Crossing theory and hierarchy mapping, IEEE Transactions on Systems, Man and Cybernetics 7 (1977), 502-523.

26. Watkins, M.E., A special crossing number for bipartite graphs: a research problem, Annals of New York Academy Sciences 175 (1970), 405-410. 